\title{
Human-Robot Interaction Control for Industrial Robot Arm through Software Platform for Agents and Knowledge Management
}

\author{
Tao Zhang, Vuthichai Ampornaramveth and Haruki Ueno
}

\section{Introduction}

At present, industrial robot arms have been widely adopted in many areas. Unfortunately, operation of them is not easy to master for workers due to complex architectures as well as various control patterns required for each situation. Therefore, if there is a user-friendly human-robot interface and through this interface workers can operate industrial robot arms by their familiar language, it will remarkably reduce the difficulty of the usage of them. The aim of this research is to develop a new human-robot interaction control approach for industrial robot arms by means of software platform for agents and knowledge management in order to construct a symbiotic human-robot system that could be adopted in industrial area (Ueno, 2002).

Conventionally, industrial robot arms only can be operated by experts who should possess sufficient knowledge on features of industrial robot arms and be able to control their movement for performing a task. To improve the human-robot interface, researchers have already developed some software interfaces for the operation of industrial robots (Mizukawa et al, 2002) (Konukseven et al, 2004) (Sales et al, 2004) (Cengiz, 2003). Unfortunately, effective use of these interfaces still depends on the technical training. This paper proposes a

c knowledge-based human-robot interaction control approach in conjunction $\circlearrowleft$ with a humanoid robot Robovie as a communication robot (Tao Zhang et al, 2005) for industrial robot arms. With this method, an operator can easily interact with the autonomous communication robot by his natural language. The communication robot transfers the sentences of the operator to a sentence parser. The key words extracted by the sentence parser are then sent to a software platform, called SPAK (Software Platform of Agents and Knowledge Management) (Ampornaramveth et al, 2004). In SPAK, it maintains sufficient knowledge on this human-robot system. According to the defined humanrobot interaction control in SPAK, industrial robot arms can be correctly oper\& ated according to operator's request. With the proposed method, a person is not required to be an expert of industrial robot arms but just an ordinary op$\bar{\Phi}$ erator of industrial robot arms. He can operate industrial robot arms like an oxpert. 
Although there are many types of industrial robot arms to be operated, the knowledge on these robots can be easily defined in SPAK in a uniform manner by using frame-based knowledge representation schema. The knowledge is maintained as a key component of a communication robot, i.e. a dialog robot, for the robot arms. Therefore, a person only needs to explain his requests to the communication robot. SPAK can assist the person to select appropriate robots and arrange their operations to satisfy the person's requests to achieve tasks. In addition, SPAK can control different types of robots even they use various kinds of operation systems. From the side of operators, it is no need to possess knowledge on the operations of different types of robots.

The remainder of this chapter is organized as follows. In section 2, humanrobot system as well as its interaction control process is modelled by framebased knowledge representation. In section 3, human-robot system is defined in SPAK according to its knowledge model using XML format. Through human-robot interaction, industrial robot arm is controlled by use of SPAK via wireless network in section 4 . Section 5 introduces an actual system comprised of human, humanoid robot (Robovie) and industrial robot arm (MELFA) and its experimental results demonstrate the effectiveness of the proposed humanrobot interaction control method.

\section{Modelling of Human-Robot System}

Human-robot interaction control for an industrial robot arm is based on the interaction between an operator of the robot arm and a communication robot. The operator's request is transferred to SPAK via wireless network and converted into commands of the robot arm. SPAK can control the robot arm with these commands and get the feedback signals from the robot. By converting these feedback signals into the sentences of natural language and speaking out these sentences by the communication robot, the operator can understand the status of the robot arm and continue his operation successfully. From this operation process, the definition of human-robot system in SPAK is one of the important components. In order to implement the definition of human-robot system, the modelling of human-robot system is necessary.

The modelling of human-robot system is based on the frame-based knowledge representation. It is well known that frame representation systems are currently the primary technology used for large-scale knowledge representation in Artificial Intelligence (AI) (Koller \& Pfeffer, 1998). A frame is a datastructure for representing a stereotyped situation (Minsky, 1974). Attached to each frame are several kinds of information, called knowledge. Collections of related frames are linked together into frame-systems. The structure of a frame is consisted of several items, such as Frame name, Frame type, A-kind-of, Descendants, Slots, etc. (Tairyou, 1998). A frame consists of slots, each of which 
has different roles in description of knowledge. Table 1 and 2 shows the definition of a frame as well as its slot.

\begin{tabular}{l|l}
\hline Items & Meanings \\
\hline Frame name & Identification for frame \\
Frame type & Type of frame \\
A-kind-of & Pointer to parent frame for expressing IS_A relation \\
Descendants & Pointer list to children frame \\
Has-part & Components of this frame \\
Semantic-link-from & Links from other frames according to their semantic relation \\
Semantic-link-to & Links to other frames according to their semantic relations \\
Slots & Components of the frame \\
\hline
\end{tabular}

Table 1.Meanings of each item in a frame

\begin{tabular}{l|l}
\hline Items & Meanings \\
\hline Slot name & Identification for slot \\
Role & Purpose of slot \\
From & Source of slot \\
Data type & Explain the attribute of information recorded into the value \\
Value & Slot value \\
Condition & Condition of slot \\
Argument & Argument for slot \\
If-required & If slot is required, check this item. \\
If-shared & If slot can be shared with other frames, check this item. \\
Frame-related & A frame related with slot \\
Frame-list-related & Several frames related with slot \\
Default & If the slot value is not determined, the default value can be \\
& recorded. But the value and default value cannot be given at \\
the same time.
\end{tabular}

Table 2.Meanings of each item in a slot

Using frames and their slots, a human-robot system can be modelled simply. This knowledge model is comprised of different frames to represent various pieces of knowledge. For instance, frames for robots include features of a communication robot and industrial robot arms as well as their operation commands. Particularly, the frames for the communication robot include the knowledge on a human-robot interface. At present, a human-robot interface can be implemented by vision recognition, robot speech, physical input, etc. While, frames for users include much information about the users. All frames for the knowledge model are organized by their ISA relations in a hierarchy. That is, a lower level frame is a subclass of its upper level frame. The bottom frames are the instances of the upper level frame. Based on these relations, a 
human-robot interaction control can be defined in frames. Table 3 illustrates a part of an example of a frame about a communication robot.

\author{
Frame: $\quad$ Communication robot \\ Type: Instance \\ A-kind-of: Robot \\ Descendants: $\quad$ Empty \\ Has-part: $\quad$ (mouth, motor, eyes) \\ Semantic-link-from: Empty \\ Semantic-link-to: Behavior \\ Table 3. A frame for communication robot
}

In the model of a human-robot system, the human-robot interaction control can be described as below. As a human operator is interacting with a communication robot, the sentences given by the operator are recognized and transferred to a software sentence parser. By this parser, the key words extracted from the sentences are sent to SPAK. In the SPAK knowledge base there exist many frames on the operation for the industrial robot arm. If the condition of an operation of the robot arm is satisfied according to the key words, the commands to the robot arm defined in this frame will be sent to the robot arm. And the robot arm will then move along the commands. After movement, the robot arm will send a feedback signal to SPAK through a software agent, which translates the feedback signal into the key words that the SPAK knowledge system can understand. As SPAK receives them, it will form some sentences corresponding to these words and send them to the communication robot. The operator can then hear the spoken sentences from the communication robot and decide the next operation.

\title{
3. Definition of Human-Robot System in Software Platform
}

\subsection{SPAK}

In order to implement a human-robot interaction control for an industrial robot arm, a software platform, called SPAK (Software Platform for Agents and Knowledge Management), has been recently developed. It is a frame-based knowledge engineering environment (Ampornaramveth et al, 2004). It provides a central module, which acts as a blackboard for comminucation channels, knowledge processing brain, memory, and does judgment, task planning and execution. It also provides software tools necessary for integration of various 
existing modules over a TCP/IP network. The features of SPAK are "platformindependent" as existing robots and software modules often rely on different platforms or operation systems, "network-aware" as the modules must interact on a network, supporting "software agent" and being "user friendly". SPAK is targeted to be the platform on which a group of coordinative robots (or their agents) operate on top of frame knowledge.

SPAK consists of the following software components:

\section{- GUI Interface:}

A user-friendly graphical interface to the internal knowledge manager and the inference engines. It provides the users direct access to the frame-based knowledge.

\section{- Knowledge Database and Knowledge Manager:}

This is the SPAK core module, which maintains the frame systems as Java class hierarchy, and performs knowledge conversion to/from XML format.

\section{- Inference Engines:}

Verify and process information from external modules, which may result in instantiation or destruction of frame instances in the knowledge manager, and execution of predefined actions.

\section{- JavaScript Interpreter:}

Interprets JavaScript code which is used for defining condition and procedural slots in a frame. It also provides access to a rich set of standard Java class libraries that can be used for customizing SPAK to a specific application.

\section{- Base Class for Software Agent:}

Provide basic functionality for developing software agents that reside on networked robots.

\section{- Network Gateway:}

This is a daemon program allowing networked software agents to access knowledge stored in SPAK. All SPAK network traffics are processed here.

\subsection{Definition of human-robot system in SPAK}

In SPAK, a human-robot system is defined according to its knowledge model by means of XML format (www.xml.com). Table 4 illustrates an example about the frame described in the XML format in SPAK for the behavior of face detection of a communication robot, which is implemented for an experiment. XML is a markup language for Internet documents containing structured information. 


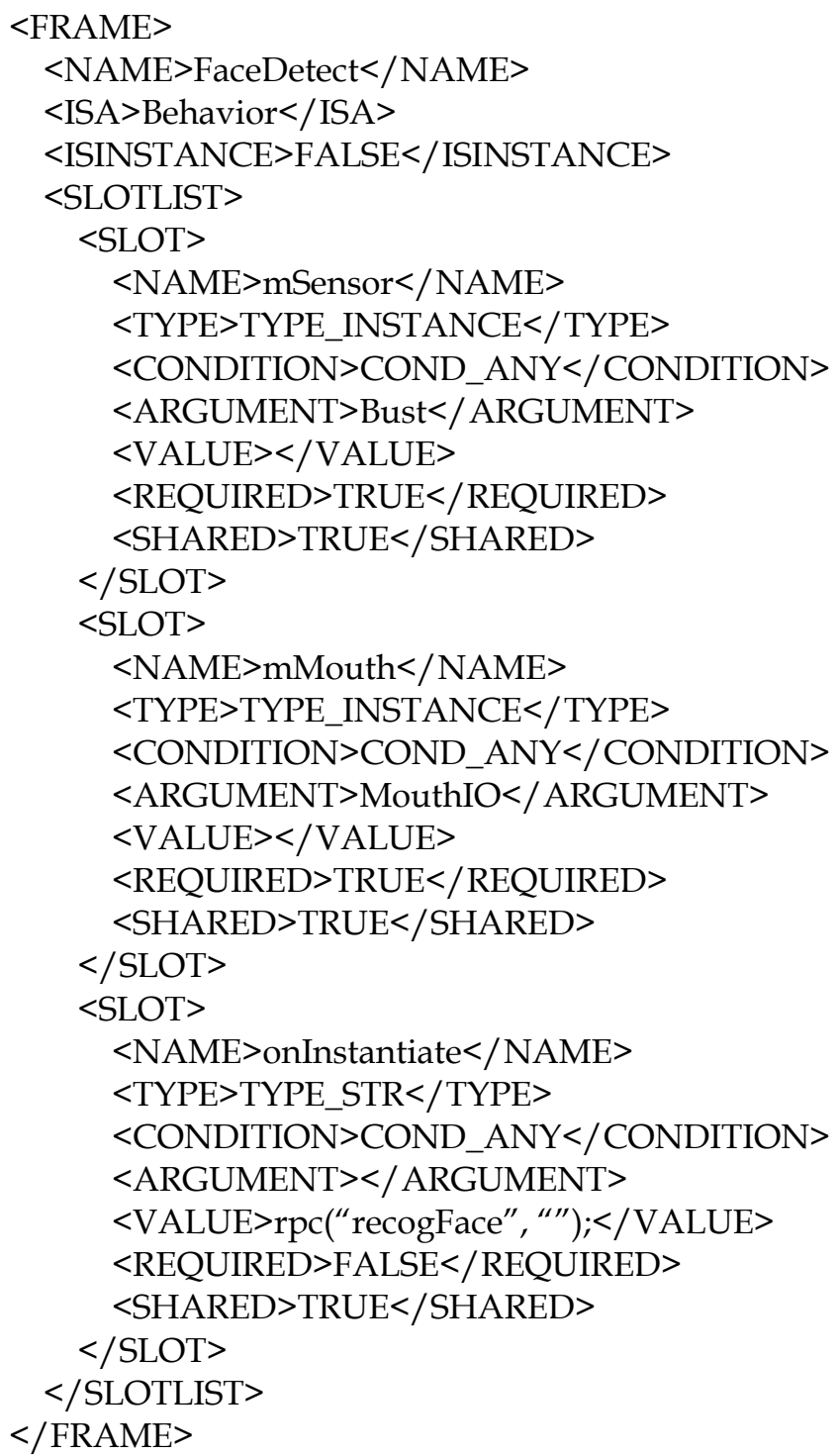

Table 4. XML format in SPAK

With XML format, frame structure as well as its contents written by slots can be defined easily. Particularly, the frame system can be illustrated in the SPAK Graphic User Interface (GUI). Besides, corresponding to the XML file, there is an interpreter to translate XML-based specifications into relative commands.

The meaning of this format can be explained corresponding to the definition of a frame in Table 1 and 2. Between <FRAME $>$ and </FRAME $>$ defines a frame. NAME refers to the frame name. ISA refers to the item of "A-kind-of". ISINSTANCE refers to the frame type. Between <SLOTLIST> and $</$ SLOTLIST> defines slots. Each SLOT gives several contents, including NAME, TYPE, CONDITION, ARGUMENT, VALUE, REQUIRED, SHARED, etc. With this XML format, a frame and its slots can be described in detail. Therefore, human-robot system can be defined in SPAK by means of XML format. 


\section{Implementation of Human-Robot Interaction Control}

In section 2, the interaction control process for an industrial robot arm has been explained. As implementing the human-robot interaction control, several technologies should be integrated and employed by SPAK. Firstly, for the interaction between an operator and a communication robot, SPAK integrates several technologies for implementing a human-robot interface, such as face detection, image recognition, speech recognition etc. Concerning face detection, by use of the robot "eyes" which are installed with video cameras, the robot can get the video image of the human's face. With the face detection program stored in the embedded computer, the robot can recognize the human by the computer vision technology. Of course, if it is the first time for this specific person, the robot will store the information about this person's face in his knowledge database by its learning function. When the robot looks this person's face again, he can recognize him at once. Thus it sends the recognition results to SPAK. Another technique that robot can use for human-robot interface is by means of speech. Since the robot system has a software program for speech recognition, SPAK just sends the recognized sentences to the communication robot, who can speak out the sentences sent by SPAK to the operator. Another important technology is to parse a natural sentence. In this humanrobot system, an operator can give his requests to the robot arm in natural language. By means of voice recognition software, such as IBM ViaVoice (http://www-306.ibm.com/software/voice/viavoice/), the sentences given by the operator can be recognized and stored into the computer. Since SPAK can generate the commands for the robot arm by several key words, it is necessary to parse the sentences and to extract the key words from them for SPAK. We have developed a simple sentence parser for SPAK using the technique of Case Grammar taking into account the features of the operations of robot arm (Bruce, 1975). With this software component, key words about objective of control, movement of robot arm, etc., will be picked out and sent to SPAK. If some inconsistencies are detected in the sentences regarding the operation of the robot arm, this parser can also generate some relative words about the inconsistencies and send them to SPAK. SPAK will ask the operator to change his commands again through the speech module of the communication robot. Although the system hierarchy has expressed the relations among frames, the execution of frame contents must be implemented in SPAK by inference engines. The inference engines used in SPAK are feedforward and backward chainings. The feedforward chaining is usually used when a new fact is added to the knowledge base and we want to generate its consequences, which may add new other facts, and trigger further inferences. The backward chaining starts with something we want to prove, find implication facts that would allow us to conclude it. It is used for finding all answers to a question posed to the knowledge base. 
Besides, the control of robots also needs their different driving programs of robots for their actions as well as some software agents to convert the signals between robots and SPAK. Particularly, for feedback control, the robot arm should be able to generate feedback signal about their status and send them to SPAK.

\section{Experiment}

\subsection{Experiment setup}

An experimental symbiotic human-robot system with human-robot interaction control for industrial robot arms has been developed, which composed of Robovie (autonomous communication robot), MELFA (industrial robot arm) and SPAK, as illustrated by Fig. 1.

Robovie was developed by Advanced Telecommunications Research Institute International (ATR) of Japan. It is designed as a kind of autonomous communication robots that can communicate and interact with human in our daily environment. It has 11 degrees of freedom (DOFs) for body motions and sensors such as an omni-directional camera, a set of pan-tilt zooming cameras, a microphone, ultrasonic range sensors, tactile sensors, etc. It can move around with its wheels. Robovie also provides many demo programs for speech, vision, motion, etc. Based on the embedded functions of Robovie, we have developed several programs for monitoring the status of all sensors and sending them to SPAK, defining many actions of Robovie as well as instructing Robovie to talk with human. 


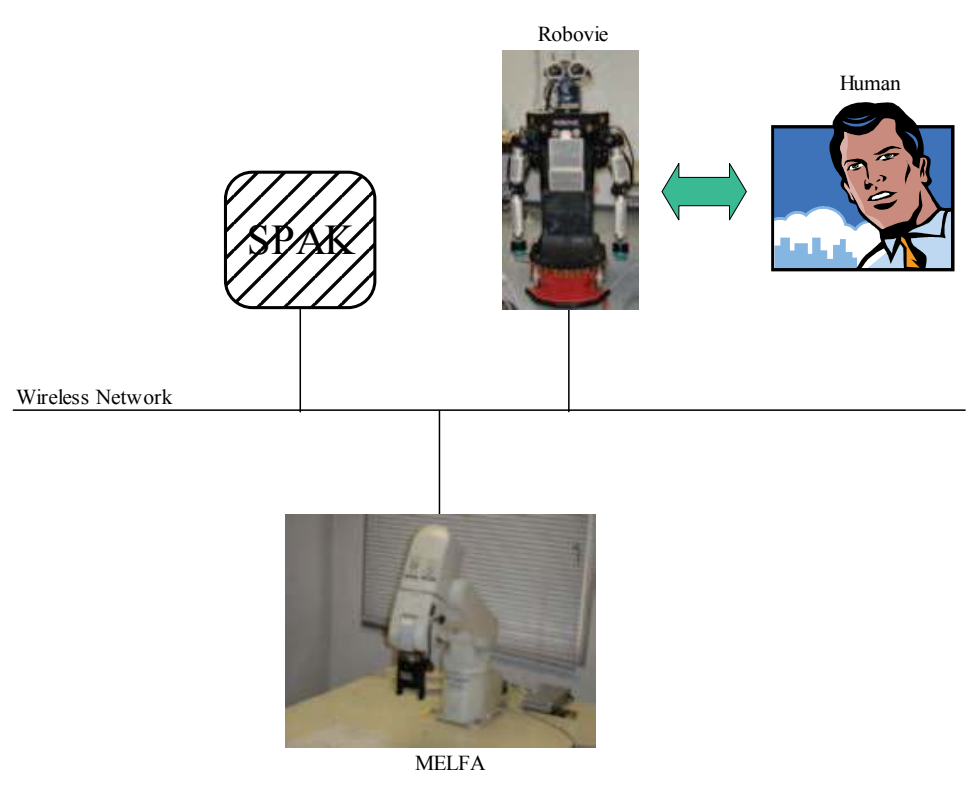

Figure 1. An actual human-robot system

Robovie can be used in the field of education, nursing, medical treatment, etc. Robovie and human can make interaction by face detection, speech and user input, so that Robovie can get the operator's commands and transfer it to SPAK.

MELFA is a kind of industrial articulated robot arm, produced by Mitsubishi Electric Co., Japan The robot arm used in this experiment has 6 DOFs generated by 6 axes. Each axis is driven by AC servomotor. The servo controller of MELFA is CR1-571. Its programming language is MELFA-BASIC IV. MELFA can move strictly along a trajectory in three-dimensional space. The tip of the robot arm can hold or release workpiece.

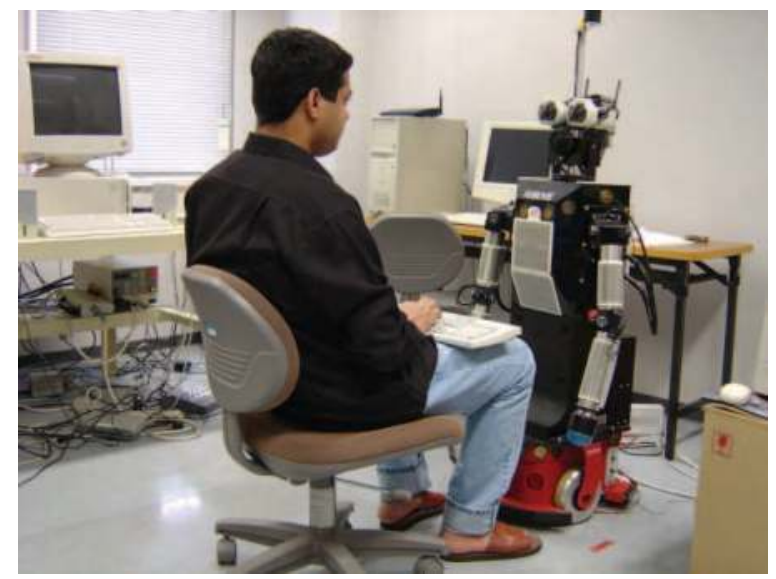

Figure 2. Environment of operation-Robovie system 
In this experiment system, Robovie, MELFA, SPAK and other software components are connected via wireless TCP/IP network. SPAK and other software components (such as vision recognition, sentence parser, software agents, etc.) are running on different computers. Therefore, this system is comprised of a distributed system. The core of this system is SPAK. Fig.2 illustrates the environment of operator-Robovie interaction for the experiment.

\subsection{Results}

Before performing the task in experiment, the human-robot system must be defined in SPAK according to the knowledge model of this system. Using the modeling method explained in section 2 the system can be modeled and defined in SPAK, as illustrated by Fig.3. From this figure, we can see that all robots and users have been defined in SPAK including their features. Each frame has the structure as Table 1 and 2 and all of them form a frame hierarchy. Fig. 4 shows the contents of frame "MELFA" which is to initiate the MELFA before operating it and Fig.5 shows the contents of frame "MELFAOn", by which the operation of turning on the power of MELFA will be executed. .

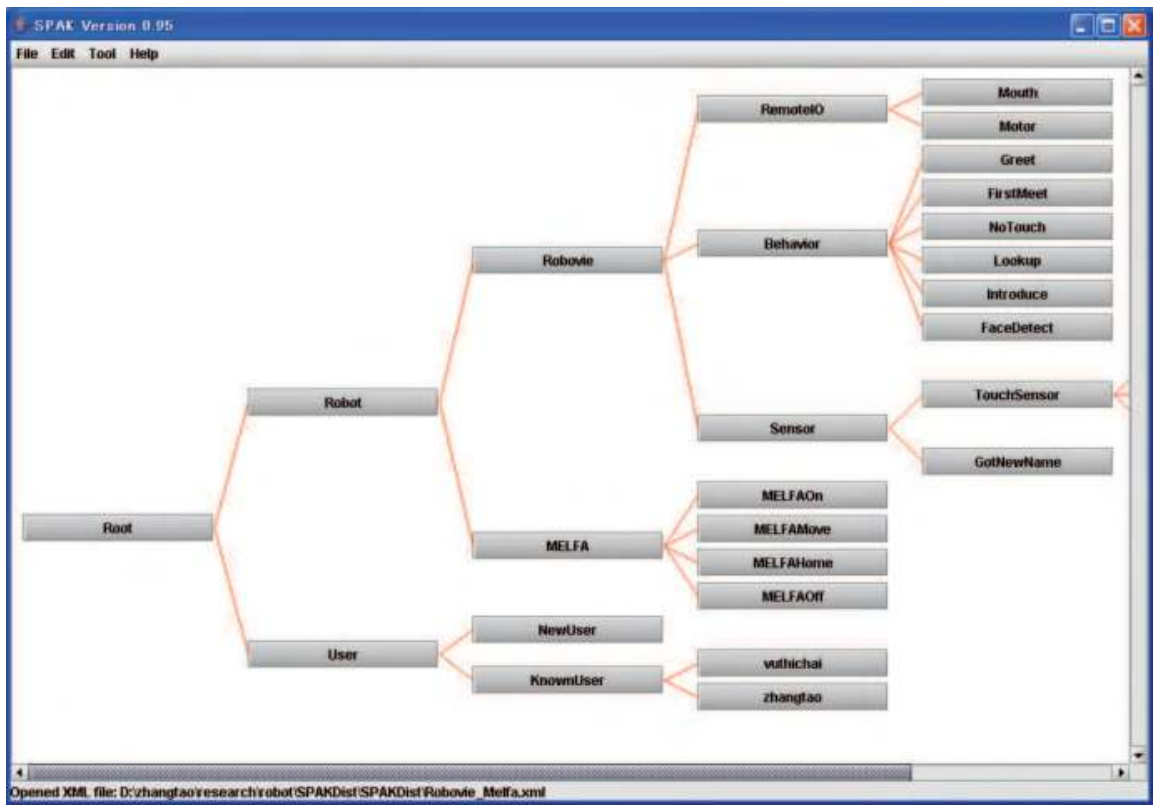

Figure 3. Interface of SPAK

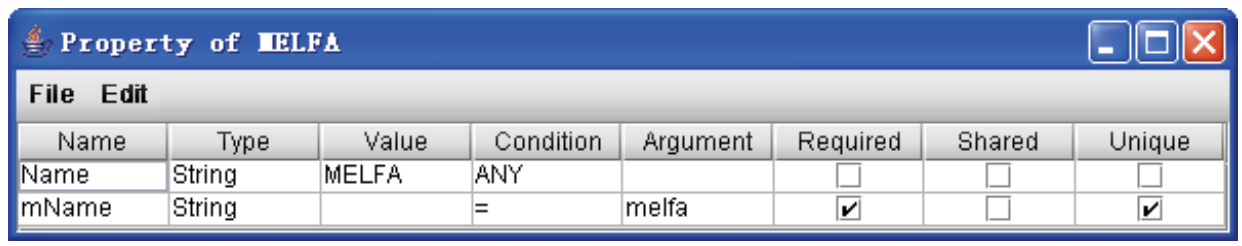

Figure 4. SPAK slot editing table for frame "MELFA" 


\begin{tabular}{|c|c|c|c|c|c|c|c|}
\hline \multicolumn{7}{|c|}{$\Leftrightarrow$ Property of IELFAOn } & $\square \square x$ \\
\hline \multicolumn{8}{|l|}{ File Edit } \\
\hline \multirow{2}{*}{$\begin{array}{l}\text { Name } \\
\text { Name }\end{array}$} & \multirow{2}{*}{$\frac{\text { Type }}{\text { String }}$} & \multirow{2}{*}{$\frac{\text { Value }}{\text { MELFAOn }}$} & \multirow{2}{*}{$\begin{array}{l}\text { Condition } \\
\text { ANY }\end{array}$} & \multirow[t]{2}{*}{ Argument } & Required & Shared & Unique \\
\hline & & & & & $\square$ & $\square$ & $\square$ \\
\hline mStatus & Instance & & ANY & MELFAOff & $\underline{V}$ & $\underline{V}$ & $\boldsymbol{V}$ \\
\hline mMouth & Instance & & ANY & Mouth & $\bar{v}$ & $\boldsymbol{V}$ & $\boldsymbol{V}$ \\
\hline mUser & Instance & & ANY & User A & $\bar{v}$ & $\bar{v}$ & $\underline{v}$ \\
\hline onInstantiate & String & melfa("SYS.. & ANY & & 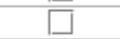 & $\boldsymbol{v}$ & $\boldsymbol{V}$ \\
\hline
\end{tabular}

Figure 5. SPAK slot editing table for frame "MELFAOn"

In Fig.6, for understanding the principle of executing frames in SPAK, the detail contents and relations of the frames of MELFA in SPAK are illustrated as an example. There exist four frames for four kinds of atomic actions of MELFA, such as "MELFAOn", "MELFAMove", "MELFAHome" and "MELFAOff". Each of them is related with the conditions expressed by the frames of "Greet", "Mouth", users and atomic actions. If the frames for the conditions are actuated at the same time, the frame with these condition frames will be actuated and the command given in the special slot "onInstantiate" in this frame will be sent to the servo controller of MELFA. MELFA will then move by the control of servo controller. For instance, the actuation of the frame "MELFAMove" is based on the actuation of the frames of "Greet", "Mouth" and "MELFAOn". With this system, we have made experiment according to the following scenario. It describes an episode of the experiment.

Robovie: (Robovie looks at the operator's face and try to recognize it.)

Robovie: Hi, how are you! I have never seen you before. What is your name?

Operator: $\quad$ (The operator types his name XXX.)

Robovie: $\quad H i, X X X$. Do you want to operate MELFA now?

Operator: Has MELFA been ready?

Robovie: $\quad Y e s$. How do you want MELFA to move?

Operator: $\quad$ I want MELFA to hold workpiece $A$ and move from $P_{0}$ to position $P_{1}$.

Robovie: $\quad$ O.K.

MELFA: $\quad$ (MELFA starts to perform the task given by the operator.)

Robovie: MELFA has finished its jobs. If you want it to perform another job, please give your command.

From the experiment, operator's commands can be correctly sent to SPAK and SPAK can convert them into the relative commands of MELFA according to the knowledge model of the system. Therefore, MELFA can perform the task correctly. Besides, for ensuring the reliability of the operation, there are some measures for safety adopted in this system. Firstly, the consistency check of the operator's sentences is installed in the sentence parser. If the operator's sentence is inconsistent with the operation of MELFA, the parser will ask operator 
to give his sentence again. Secondly, SPAK provides some frames for confirmation. Before performing the movement of MELFA, SPAK will instruct Robovie to repeat the task given by operator again. If operator confirms its correctness, MELFA will be controlled to move. If there exist any problems, SPAK will instruct Robovie to tell operator by speech as soon as possible. Thirdly, MELFA also has some measures to protect itself. With this system, the error information generated by MELFA can be also transferred to SPAK and spoken out by Robovie.

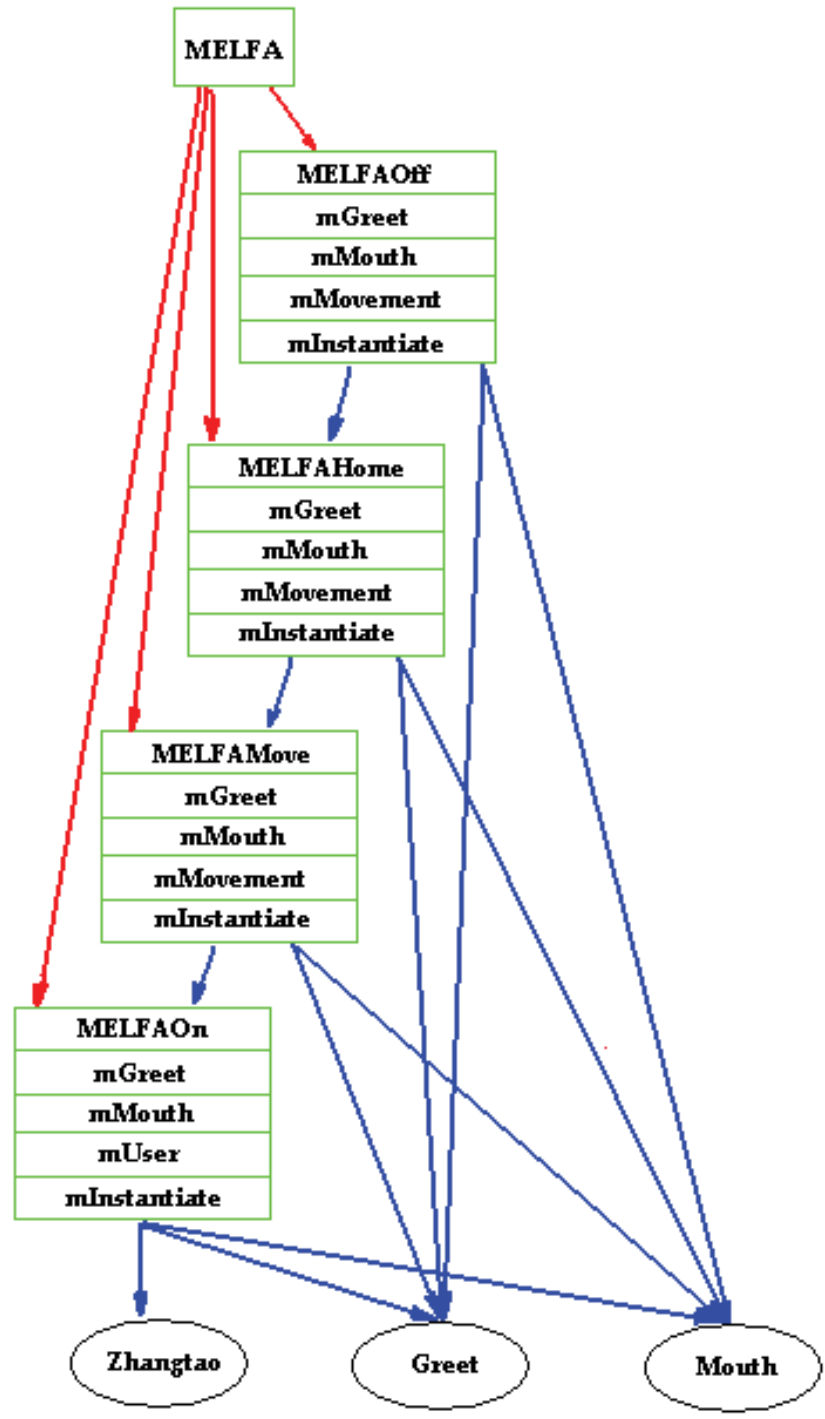

Figure 6. Frames for the operation of MELFA in SPAK 


\section{Discussions}

\subsection{Flexibility and extensibility of the proposed human-robot interative control method}

Since the proposed human-robot interaction control method is based on the knowledge model and implemented by SPAK, it has strong flexibility and extensibility. The flexibility of the proposed method is reflected by that, in the knowledge model it is possible to define various kinds of frames to control robot behaviors for different tasks based on human requests. SPAK can integrate many software components to support the implementation of human-robot interaction control for industrial robot arms. The proposed method separates the human-robot interaction control of robots into two stages: designing the knowledge model and implementation in the SPAK. It is possible to adopt same ways (same robots) to implement different tasks or adopt different ways (different robots) to implement same tasks. Therefore, high-level knowledge model design or low-level software component development in SPAK provides a large space to flexibly that performs the tasks for human requests.

The extensibility of the proposed method is resulted from the following several points of views. This method is open to many types of robots. Any new types of robots can be described by the frames in the knowledge model and integrated into the SPAK. The behaviors of multiple robots can be easily combined for a specific task. Different combinations can extend the ability of the system to serve to human. Except natural language, many types of humanrobot interaction can be integrated by the proposed method. With the increase of the complexity of tasks, the control performance of the proposed method can be improved by extending the functions described in the knowledge model and implemented by SPAK.

\subsection{Knowledge management and improvement by learning in SPAK}

Due to the increase of the knowledge required for human-robot system, the knowledge management and improvement becomes more and more important. This is the current research topic for improving the proposed method and realizing the goal of constructing a highly intelligent symbiotic human-robot system in the future. The knowledge management includes the organization and optimization of knowledge. Although a frame and its slots provides strong ability to describe various types of objects and frame hierarchy can organize the frames into a system, it needs mechanism to classify the different types of frames, renew the contents of frames, delete the useless frames, detect the logical errors among frames, and so on. Currently, an expert manages the knowledge manually for a human-robot system. It is definitely needed to de- 
velop functions to automatically fulfill these kinds of knowledge management. To imitate human ability, learning new knowledge by means of SPAK for improving the knowledge model is being developed in our research. At present, the learning function is implemented as a Java-based program and developed based on the interaction oriented learning approach. It can exchange information with SPAK and create new frames in SPAK. During the process of interaction, a user will teach a communication robot how to perform a robot behavior or accomplish a specific task by various behaviors for different robots. According to user's teaching, new strategy frames can be created in SPAK by the learning function. The learning function performs a searching process in SPAK. New strategy frames are created in SPAK when the relative behavior frames or strategy frames for robot behaviors are found. The searching route is according to the types of robots and their titles of frames. Since SPAK is a Javabased software system and it consists of Java classes to deal with the manipulation of frames, it is therefore easy to create frames and put them into the new strategy frames in SPAK. If SPAK can not find the existing behavior frames or strategy frames, it will ask the user to provide more information about the new behaviors of robots and therefore create new frames in SPAK. Since this learning function is still under development, it will be continuously improved by the further research.

\subsection{Future issues}

In order to realize the final goal to construct a highly intelligent symbiotic human-robot system, concerning the proposed control method there still exist many attractive, challengeable issues needed to solve. As mentioned above, the knowledge model adopted by the proposed method needs to be improved on its management. We want to combine the proposed method with other kinds of control approaches to improve the control performance for a symbiotic human-robot system, such as adaptive control, robust control, fuzzy control, etc. In addition, the reliability of the proposed human-robot interaction control of robots is needed to continuously improve. We will apply this method for more different typical tasks in wide areas. With the improvement of robot functions in the near future, the proposed method will be able to adopt for more real applications. 


\section{Conclusions}

A new human-robot interaction control approach for industrial robot arm was proposed. In this method, operator's requests can be directly obtained by an autonomous communication robot through human-robot interaction, and converted into control instructions for industrial robot arms by SPAK. In addition, the status of the robot arm can be obtained by SPAK and informed to the operator by the speech function of the communication robot. Based on this method, an experimental symbiotic human-robot system for industrial robot arms was constructed and evaluated. The experimental results demonstrate the effectiveness of the proposed method. The proposed idea of human-robot interaction control approach for industrial robot arms has strong flexibility and extensibility. It can be extended to construct many kinds of symbiotic human-robot systems including different kinds of robots. The knowledge management and improvement can be realized by learning functions in SPAK. In the future research, more issues will be considered to solve and the control performance of human-robot system will be further improved. We believe that the symbiotic human-robot system with human-robot interaction control has great potentials for future human society. 


\section{References}

Ampornaramveth, V.; Kiatisevi, P. \& Ueno, H. (2004). SPAK: Software Platform for Agents and Knowledge Management in Symbiotic Robotics. IEICE Trans. Information and Systems, Vol. E87-D, No. 4, pp. 886-895.

Bruce, B. (1975). Case systems for natural language. Artificial Intelligence, Vol. 6, pp. 327-360.

Cengiz, M. C. (2003). Software development for man-machine interface for an industrial robot. Thesis of master degree.

Koller, D. \& Pfeffer, A. (1998). Probabilistic frame-based systems. Proc. of the 15th National Conference on AI (AAAI-98), pp. 580-587.

Konukseven, E. L. \& Abidi, A. (2004). Developmen of man machine interface software for an industrial robot. Proc. of 2004 IEEE Symposium on Virtual Environment, Human-Computer Interfaces and Measurement Systems, (VECIMS), pp. 49-53.

Minsky, M. (1974). A Framework for representing knowledge. MIT-AI Laboratory Memo 306.

Mizukawa, M.; Matsuka H., et al, (2002). ORiN: open robot interface for the network - the standard and unified network interface for industrial robot applications. Proceedings of the 41st SICE Annual Conference, Vol. 2, pp. 925-928.

Sales, J.; Fernandez, R., et al, (2004). Telecontrol of an industrial robot arm by means of a multimodal user interface: a case study. Proceedings of 2004 IEEE International Conference on Systems, Man and Cybernetics, Vol. 1, pp. 76-81.

Tairyou, G. (1998). Development of frame-based knowledge engineering environment ZERO. Master Thesis, Tokyo Denki University.

Tao Zhang, H. Ueno (2005), A Frame-Based Knowledge Model for Heterogeneous Multi-Robot System, IEEJ Trans. EIS, Vol.125, No.6, pp.846-855.

Ueno, H. (2002). A knowledge-based information modeling for autonomous humanoid service robot. IEICE Transactions on Information and systems, Vol. E85-D, No. 4, pp. 657-665. 


\section{Industrial Robotics: Theory, Modelling and Control \\ Edited by Sam Cubero}

ISBN 3-86611-285-8

Hard cover, 964 pages

Publisher Pro Literatur Verlag, Germany / ARS, Austria

Published online 01, December, 2006

Published in print edition December, 2006

This book covers a wide range of topics relating to advanced industrial robotics, sensors and automation technologies. Although being highly technical and complex in nature, the papers presented in this book represent some of the latest cutting edge technologies and advancements in industrial robotics technology. This book covers topics such as networking, properties of manipulators, forward and inverse robot arm kinematics, motion path-planning, machine vision and many other practical topics too numerous to list here. The authors and editor of this book wish to inspire people, especially young ones, to get involved with robotic and mechatronic engineering technology and to develop new and exciting practical applications, perhaps using the ideas and concepts presented herein.

\section{How to reference}

In order to correctly reference this scholarly work, feel free to copy and paste the following:

Tao Zhang, Vuthichai Ampornaramveth and Haruki Ueno (2006). Human-Robot Interaction Control for Industrial Robot Arm through Software Platform for Agents and Knowledge Management, Industrial Robotics: Theory, Modelling and Control, Sam Cubero (Ed.), ISBN: 3-86611-285-8, InTech, Available from: http://www.intechopen.com/books/industrial_robotics_theory_modelling_and_control/humanrobot_interaction_control_for_industrial_robot_arm_through_software_platform_for_agents_and_kn

\section{INTECH}

open science | open minds

\section{InTech Europe}

University Campus STeP Ri

Slavka Krautzeka 83/A

51000 Rijeka, Croatia

Phone: +385 (51) 770447

Fax: $+385(51) 686166$

www.intechopen.com

\section{InTech China}

Unit 405, Office Block, Hotel Equatorial Shanghai

No.65, Yan An Road (West), Shanghai, 200040, China 中国上海市延安西路65号上海国际贵都大饭店办公楼 405 单元 Phone: +86-21-62489820

Fax: +86-21-62489821 
(C) 2006 The Author(s). Licensee IntechOpen. This chapter is distributed under the terms of the Creative Commons Attribution-NonCommercial-ShareAlike-3.0 License, which permits use, distribution and reproduction for non-commercial purposes, provided the original is properly cited and derivative works building on this content are distributed under the same license. 